\title{
Three Patterns of Motion Which Change the Perception of Emotional Faces
}

\author{
Alhadi Chafi ${ }^{1,2}$, Loris Schiaratura ${ }^{1,2}$, Stéphane Rusinek ${ }^{1,2}$ \\ ${ }^{1}$ Université de Lille-Nord de France UDL3, Villeneuve d'Ascq, France \\ ${ }^{2}$ Laboratoire PSITEC, Villeneuve d'Ascq, France \\ Email: chafi@live.co.uk
}

Received October $1^{\text {st }}$, 2011; revised November $5^{\text {th }}$, 2011; accepted December $7^{\text {th }}, 2011$

\begin{abstract}
The aim of the study was to focus on the relationship between motion and emotion. Relying on studies in Behavioral Neurology and Social Psychology, it is believed motion is one of the core components of Emotion. The study uses basic emotional faces (i.e., Happy, Surprised, Fearful, Sad, Disgusted and Angry) which are presented displaying patterns of motion (i.e., a Parabolic motion, a Translational motion and a Wave-like motion). Hypotheses are that the wave-like motion will increase perceived intensities and arousal related to positive emotional faces (i.e., Happy and Surprised), and simplify their recognition. Otherwise, the parabolic motion is hypothesized to increase perceived intensities and arousal related to negative emotional faces (i.e., Angry, Disgusted, Fearful and Sad), while enhancing their recognition. Results showed that "Happy" is the most recognized face and "Fearful" is the least recognized one. Concerning Perceived Intensity, an Emotional Face main effect and an Interaction Motion Pattern $\times$ Emotional Face were obtained. Finally, the Arousal dimension yielded two main effects, one for the Emotional Face and one for the Motion Pattern. On one hand, results we found are very promising in understanding the part played by motion in Arousal. On the other hand, further research still has to be done so as to question the exact effects of the Translational, Parabolic and Wave-like motion patterns, especially in more dynamic contexts.
\end{abstract}

Keywords: Emotional Face; Motion Pattern; Self-Reports; KDEF

\section{Introduction}

The relationship between motion and emotions has always been a matter of investigation in the way that motion, per se, is believed to involve emotional experience. For instance, some authors demonstrated that certain patterns of motion have more emotional potential than others (Rimé, Boulanger, Laubin, Richir \& Stroobants, 1985; Rimé \& Schiaratura, 1991). A similar range of studies showed that very simple patterns of motion from an object involve animation and emotional attributions (e.g., Heider \& Simmel, 1944). Henceforth, the aim of the present study is to show that three specific patterns of motion from a disk, in which was inlaid a facial expression, will have an effect on the recognition of this latter and on the perceived intensity and arousal related to that emotional face. Indeed, theories of the embodied cognition (e.g., Barsalou, 1999) gave rise to findings which clearly show congruency effects between certain types of executed actions, or even simulated actions, and experienced emotional dynamics. These congruency effects could be illustrated as following: an approach movement (e.g., pulling a lever) was faster when positive feelings were experienced whereas an avoidance movement (e.g., pushing a lever) was faster when negative feelings were experienced (Alexopoulos \& Ric, 2007; Brouillet, Heurley, Martin, \& Brouillet, 2010). Similarly, Freina, Baroni, Borghi, and Nicoletti (2009) showed that participants were faster in reaching an away button when reading positive words whereas they were faster in pulling their hand back to their body when reading negative words. At a perceptual level, Podevin (2009) demonstrated that three motion patterns were linked to emotions, namely, the Translational, Parabolic and Wave-like motion patterns. For instance, Rusinek (2009) used the Wave-like motion in order to desensitize an arachnophobia in a sub-clinical population. Participants of this experiment could bring towards them a false spider closer than the other sub-clinically arachnophobics who were not exposed to the Wave-like motion/picture of a spider association. According to Podevin (2009), the Parabolic motion enhanced memory for negative words whilst the Wave-like motion enhanced memory for positive words. Tremoulet and Feldman (2000) showed that the perception of a parabolic motion from an object (i.e., a circle or a cylinder) gave rise to animation attribution to the object in an adults sample. Finally, the Translational motion is evaluated by a sample of adults and children as neutral or weakly positive (Podevin, 2009). The positive triggering tendency of an horizontal translational stimulus was also supported by studies of Phaf and Rotteveel (2009). These authors showed that a left-to-right arrow gave rise to faster approach movements and more positive evaluations than a right-to-left arrow in Dutch left-to-right readers. Yet, as the presented arrow was static, it is assumed that it did not have the same effects as the Translational motion, which was assessed as neutral. Therefore, the investigation consisted in answering how these three specific patterns of motion from a single object will have an effect on the recognition of still emotional faces depicting the six basic emotions and on their related emotional assessments. Hypothesis is that the Wave-like motion will be related to positive emotional faces whereas the Parabolic motion will be related to negative emotional faces. Concerning the 
Translational motion, it will have a medium effect on both types of emotional faces.

Facial expressions of emotion have long been studied in Psychology and Neuroscience fields (Ekman \& Friesen, 1976; Ekman, 1993; Ekman, 1999 Adolphs, 2002), but most research in the domain are interested in still images (Calvo \& Lundqvist, 2008; Goeleven, De Raedt, Leyman, \& Verschuere, 2008). Furthermore, Batty and Taylor (2003) showed that the processing of emotional faces is automatic and rapid for the six basic emotions (i.e., sadness, fear, disgust, anger, surprise and happiness). Ambadar, Schooler and Cohn (2005) showed that motion in a face, for the six above-mentioned emotional expressions, was the most important feature to be able to decipher the expressed emotion. Thus, it is thought to be important to study the six basic emotional expressions along with the three patterns of motion which are believed to be linked to emotions.

There are two kinds of approaches in the Motion Research: 1) biological motion field, which refers to the identification and recognition of a biologically possible motion, often done by a real being (i.e., the flexion/extension of an arm, etc.); or 2) nonbiological motion field, which does not refer to any living being and does not need to be biologically possible (i.e., the bouncing of a ball, etc.). Many research have shown a link between motion and emotion, whether this motion is biological (Johansson, 1973; Blake \& Shiffrar, 2007) or non-biological (Heider \& Simmel, 1944; Michotte, 1946/1962).

\section{Biological Motion Research}

Johansson (1973) has been the first author in this field of interest. In fact, he placed dots on the joints of human bodies who were filmed in the darkness. He then exposed his participants to the moving dots only, in order to prevent his participants to recognize a human structurally, and he showed that participants are still able to recognize a running, dancing or walking individual. That study was the starting point of a long tradition in the Motion Research. For instance, Cutting and Kozlowski (1977) showed that the perception of moving dots is sufficient to identify friends. Troje, Westhoff, and Lavrov (2005) showed that deprivation of structural information, such as size or shape, does not have an effect on the identification and recognition of individuals as participants essentially rely on the kinetics of individuals' walk. In addition, Troje and Westhoff (2006) showed a kind of inversion effect related to the very specific position of feet in terrestrial animals. These authors also postulated the existence of a "Life Detector" in the visual brain system of the evoked animals. This latter hypothesis is also supported by works of Méary, Kitromilides, Mazens, Graff and Gentaz (2007) who showed that 4-days-old human infants looked longer at non-biological motion compared to biological motion. That finding suggests that infants motion perception was already attuned to biological kinematics.

Thereby, everything coming from the Biological Motion Research seems to suggest that motion is at least as, if not more, important as still features of an individual for its recognition and identification. It is now possible to wonder about the nonbiological motion.

\section{Non-Biological Motion Research}

The ranges of interests of Psychologists had first highlighted non-biological motion rather than motion from individuals or animals. For example, Michotte (1946/1962) studied what he called "phenomenal causality", which can be defined as the fact that participants evoked interactions between the moving objects, even though they knew that these objects were inanimate. Heider and Simmel (1944) found that depending on the random motion patterns of different objects (e.g., a triangle, a circle, etc.), participants attributed different intentions, attitudes and emotions to the moving objects (i.e., anthropomorphism). This anthropomorphism can be defined as the fact of giving humanlike emotional and behavioral properties to the moving objects, with the description of their patterns of motion as a base to assess these attributes. Rimé, Boulanger, Laubin, Richir and Stroobants (1985) showed that an intercultural consensus between Belgians, Americans and Zairians existed for 3 out of 5 kinetic structures. In that study, it was clear that it was not the shapes of the presented objects that were important in the involvement of emotional perception but it was rather the kinetic structure of motion. In a latter research, Tremoulet and Feldman (2000) showed that a parabolic motion from a white single object (i.e., a cylinder or a circle) on a dark background can give rise to a perception of animation. That perception is induced by combined changes of speed and direction, and again, that result had been found by Michotte fifty years before. Nevertheless, the groundbreaking point of Tremoulet and Feldman dwells in the use of a specific pattern of motion, namely, the parabolic motion. Here, it is noteworthy to refer to Casasanto and Dijkstra (2010) who defined three dimensions related to emotions: verticality, horizontality and flexion/extension. Concerning the first dimension, it seems that up movements of the arm favour positive statements whereas down movements favour negative statements. Concerning the horizontality dimension, Phaf and Rotteveel (2009) showed that Dutch left-to-right readers evaluated more easily an arrow in the reading direction (i.e., from left-to-right) than an arrow in the opposite direction (i.e., from right-to-left). Moreover, they experienced positive emotions when exposed to the left-to-right arrow whether the evaluation task was explicit (i.e., a self-report scale) or implicit (i.e., arm flexion vs. arm extension). Also, Alexopoulos and Ric (2007), based on Chen and Bargh (1999), showed that the flexion of an arm is faster when exposed to happy words whilst the extension of an arm is faster when exposed to sad words. All these findings showing concruency effects between a dimension and emotional processes could be explained in terms of the affective monitoring hypothesis (Phaf \& Rotteveel, 2009). According to this assumption, certain perceptual features would guide our emotional system towards corresponding affective processes, namely: 1) Verticality: an "up" stimulus would be related to positivity whereas a "down" stimulus is believed to be related to negativity; 2) Horizontality: a left-to-right stimulus would be seen as more positive than a right-to-left stimulus in a left-to-right reader; and 3) Flexion/Extension: the flexion of an arm is faster during the presentation of a positive stimulus whilst the extension of an arm is faster during the exposure to a negative stimulus. The same type of congruency effects were found for motion perception by Podevin (2009) and Rusinek (2009) as above-mentioned. Effectively, these authors showed that a wave-like motion is linked to positive emotions whilst a parabolic motion is linked to negative ones. They also postulate that a translational motion is related to a neutral valence. Therefore, it is noteworthy to discuss the occurrence and type of such a congruence in the relationship between motion and emotion. 


\section{The Motion-Emotion Congruence: Evidence from 3 Patterns of Motion}

Recently, Podevin (2009) investigated the effect of a motion from a dark circle on a white background on the affective state of both adults and children. According to Podevin, there is a link which readily seems to follow a "congruence system" for three specific patterns of motion (see Figure 1). Thus, Podevin showed that a parabolic motion pattern was mainly assessed as emotionally negative while a wave-like motion pattern was strongly evaluated as positive. Furthermore, individuals attributed a neutral valence to a translational motion pattern. At a cognitive level, the Parabolic motion enhanced memory for negative words whereas the Wave-like motion ameliorated memory for positive words in an adults sample (Podevin, 2009). Another groundbreaking finding was that the Parabolic motion induced a deceleration in a writing-speed task, what is marked by a negative emotional dynamics (Natale \& Hantas, 1982). These associations, namely Wave-like/positive emotions, Parabolic/negative emotions, Translational/neutral condition were replicated in children aged from 9 to 12 years old, both at a perceptual and cognitive levels (Podevin, 2009). Therefore, not only the emotional perception is affected by the motion patterns displayed according to participants' evaluations, but this relationship has an impact on cognitive ressources (i.e., the memory of emotional words) and operations (i.e., a writing-speed task). In line with this congruence hypothesis, Rusinek (2009) used the Wave-like motion in an arachnophobia desensitization on a sub-clinical sample. That latter study empowers the assumption that the Wave-like motion is linked to positive emotional processes.

In the present study, congruency effects between patterns of motion and recognition and perceived intensity of emotional faces are expected. It is assumed that the Parabolic motion will increase the recognition and perceived intensity of negative faces (i.e., Sad, Fearful, Disgusted, and Angry) while the Wave-like motion will increase the recognition and perceived intensity of positive faces (i.e., Happy and Surprised) compared to the Translational motion.

For the arousal dimension, no specific hypothesis was postulated as this measure for the above-mentioned patterns of motion has never been done before.

\section{Method}

\section{Participants}

Sixty-one French students (48 women and 13 men) from the University of Lille North of France UDL3 were randomly recruited with the only criterion of having a normal or correctedto-normal vision. All of them are right-handed people and native French speakers.

\section{Material and Apparatus}

\section{Emotional Faces}

According to Goeleven, De Raedt, Leyman and Verschuere (2008), the Facial Action Coding System (FACS; Ekman \& Friesen, 1976) is nowadays old-fashioned and limits ecological validity. Other sets of stimuli that could be possibly used to question our hypotheses are: the Japanese and Caucasian Facial Expressions of Emotion (JACFEE; Matsumoto \& Ekman, 1988) and the Montreal Set of Facial Displays of Emotion (MSFDE;
Beaupré, Cheung, \& Hess, 2000). Yet, the weak number of pictures these sets contain still remains a substantial limitation. Therefore, Goeleven et al. (2008) measured the emotion, perceived intensity, and arousal ratings for 490 frontal pictures from the Karolinska Directed Emotional Faces (KDEF; Lundqvist, Flykt, \& Öhman, 1998). Arousal is one of the most relevant components of emotion as it directly impacts on motivetional dimension (Lang, Greenwald, Bradley, \& Hamm, 1993). The study of Goeleven et al. both confirms the validity of the KDEF and offers many interesting results. Effectively, it appears that "Happy" is the best recognized emotional face while "Fearful" is the least recognized one. Concerning the intensity dimension, "Disgusted”, "Happy”, and "Surprised" are the most intense emotional expressions. The arousal dimension shows no differences between emotional categories, but there are discrepancies between emotional faces and neutral faces. Although this research is in line with previous emotion studies (Gross \& Levenson, 1995), it has the limitation of only including female participants. Nevertheless, the procedure they used so as to assess emotions of the participants via self-reports is the same as we are using here.

In the present study, faces were chosen on the criteria of recognition, perceived intensity of emotion and arousal ratings. Thus, participants were exposed to strongly recognizable faces involving high ratings of perceived intensity and arousal, all of which reflecting a high emotional experience. Hereby, six facial expressions of emotions (i.e., Happy, Fearful, Angry, Surprised, Sad and Disgusted) were retained among these validated by Goeleven et al. (2008). The "gender of actor" factor was controlled by only showing male emotional faces (see Figure 2).

\section{Patterns of Motion}

The disk's size is exactly the same in this study as in the experiments of Podevin (2009) and Rusinek (2009). It precisely has a diameter of 4.1 centimeters. Effectively, that surface covers a large enough space so that a face is seen without disturbing the participants. Background is white and the emotional face is encrusted in the disk.

Concerning trajectories, the Translational motion does not seem to disrupt cognitive resources of an individual and a schematic facial expression of smile is associated to that motion by $20 \%$ of children. The Parabolic trajectory would be able to increase cognitive resources directed towards negative emotional

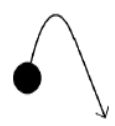

(a)

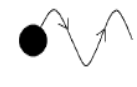

(b)

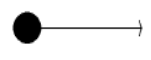

(c)
Figure 1.

Patterns of Motion: (a) Parabolic, (b) Wave-like, (c) Translational.
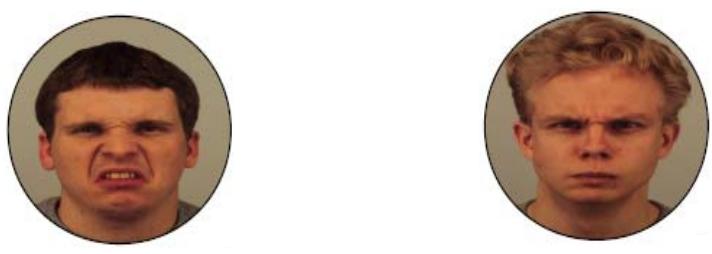

Figure 2.

Pictures of "Disgusted” (left) and "Angry" (right) emotional faces as they were presented. 
stimuli. Finally, the trajectory of the Wave-like motion gathering spring, pendulum and translation is associated to positive emotional processes and a smile is linked to that motion by $60 \%$ of children (see Podevin, 2009 for a review).

Motion patterns lasted 5 seconds for any trajectory. Therefore, we showed 18 associations (3 Motion Patterns $\times 6$ Emotional Faces) to each participant. Orders of the associations were counterbalanced ( 3 orders at last) so that we eliminated the order effects.

\section{Measurements}

In order to assess self-reported emotions, every participant was given a brochure composed of one forced-choice question for the emotion recognition task and two items before watching the whole presentation. The two items are respectively: an Intensity item taken from the Intensity of Emotion Scale (IES; Schaefer, Sanchez, Nils, \& Philippot, 2010) and an Arousal item taken from the Self-Assessment Manikin (SAM; Lang, 1980). The brochure is made of 19 pages (including a cover page with a short instruction) as participants were shown 18 slides. Thus, each slide was rated on the recognition question and the two scales.

For the emotion recognition task, participants were asked to surround the word that matches the expressed emotion. Proposals were each of the six basic emotions to which we added "Neutral” and "?” (Indistinct). Concerning the IES (ranging from 1 = "not intense at all" to 9 = "completely intense"), participants were asked to surround the number on the scale that best represents the intensity of the emotion they chose in the recognition task. Finally, the SAM (ranging from 1 = "calm" to 9 = "excited") consists in graphic depictions of various stages of arousal, and the participants had to answer the question "What did you feel when viewing the face? Match the graphic that corresponds to your state. At one extremity of the scale, you are excited, awaken, stimulated; at the other extremity, you are relaxed, unstimulated, calm”.

\section{Procedure}

Each participant came individually in a box of the PSITEC Lab, located in the Department of Psychology. The experimenter made recruitment by asking students if they had about 10 minutes to assess emotional faces. Following the entering of the box, instructions could be said:

"You are to assess emotional faces via a brochure. Each page of that brochure corresponds to one face. You will plainly follow the instructions on the screen and in the brochure when engaged in the task. First, we are going to make a bit of relaxation”.

After ensuring that the participant is ready for the relaxation phase, the experimenter asked the participant to close his eyes, to relax every muscular group, including his face, and to deeply and regularly breathe. Then, he asked individuals to perform arm and leg flexions while still deeply breathing. Relaxation lasted about 2.5 minutes. When the relaxing sequence was finished, the participant was informed that he could begin by pressing any key. Experimenter then asked him to follow carefully instructions given on the screen. Displays were seen on a 17', inches screen and were created via Microsoft PowerPoint 97-2003. Each participant saw every type of emotional face with every type of motion pattern (i.e., 18 emotional displays). Between every emotional display, a transition slide instructed the participant to fill in the corresponding page and then press any key to see the next display. This brochure consisted in "paper-pen” tests, so pens were available to participants. Finally, a dehoaxing step was made in the following manner:

"As you could have noticed, you saw many times the same emotional faces. In fact, these faces were associated to different motion patterns. The patterns of motion you saw are believed to be differently associated to emotions, according to recent studies in that field.”

\section{Results}

A 3 (Motion Pattern) $\times 6$ (Emotional Face) ANOVA was performed using within-subjects designs for each dependent variable, namely, the Arousal, the Recognition and the Perceived Intensity.

\section{Self-Assessed Arousal}

The analysis revealed a main effect of Motion Pattern, $F$ (2, $120)=5.24 ; p<.01$, indicating that the Wave-like motion $(M=$ 3.33, $S D=1.91)$ was significantly more arousing than the Translational $(M=3.14, S D=1.76)$ and Parabolic $(M=3.10$, $S D=1.80)$ motion patterns.

Concerning the Emotional Face factor, another main effect was found, $F(5,300)=3.15 ; p<.01$. This effect indicated that "Sad" $(M=2.81, S D=1.55)$ was significantly less arousing than "Disgusted" $(M=3.38, S D=1.80)$, "Happy" $(M=3.37$, $S D=2.05)$, "Angry" $(M=3.28, S D=1.91)$, and "Fearful” $(M=$ 3.23, $S D=1.82$ ).

Even though the Motion Pattern $\times$ Emotional Face interacttion was not significant, $F(10,600)=.97 ; p=.46$, our a priori hypotheses permitted us to look into Post-hoc tests. These comparisons showed us that the "Happy" face displaying the Wave-like motion ( $M=3.71 ; S D=2.11$ ) was more arousing than when associated with the Parabolic motion $(M=3.21$; $S D$ $=1.95), p<.02$, or the Translational motion $(M=3.20 ; S D=$ 2.09), $p<.02$. Another finding was that the "Sad" face was more arousing when associated with the Translational motion $(M=2.97 ; S D=1.67)$ than when associated with the Parabolic motion $(M=2.53$; $S D=1.47), p<.04$.

Therefore, it seems that Self-Assessed Arousal was modulated by Motion Patterns for "Happy" and "Sad" faces only. Analysis demonstrate that "Happy" face was affected by the most arousing motion (i.e., the Wave-like motion) whereas "Sad" face was affected by the least arousing one (i.e., the Parabolic motion). These results are congruent regarding the main effects of Emotional Face and of Motion Pattern taken together. Recognition and perceived intensities of emotional faces could help explaining these findings. (see Table 1)

\section{Recognition Rate}

The analysis revealed a main effect of Emotional Face, $F$ (5, $300)=22.30, p<.0001$. Table 2 shows the average frequencies means and standard deviations for each emotional face. The Emotional Face effect indicated that "Happy" $(M=.98 ; S D$ $=.15$ ) was more recognized than any other face except the "Disgusted" one $(M=.90 ; S D=.29)$, which was itself more recognized than "Sad" $(M=.76 ; S D=.44)$ and "Fearful" $(M$ $=.51 ; S D=.50)$. The very same results as those for "Disgusted" were obtained for "Surprised" $(M=.88 ; S D=.33)$. "Fearful" and "Sad" were found to be, respectively, the least and second-least recognized Emotional Faces. 
The main effect of Motion Pattern was not significant, $F$ (2, $120)=2.27, p=.11$. However, further tests showed that the Parabolic motion $(M=.82 ; S D=.34)$ induced more Recognition than the Wave-like motion $(M=.79 ; S D=.36)$.

Even though the Motion Pattern $\times$ Emotional Face interacttion was not significant, $\mathrm{F}(10,600)=1.51, p=.13$, further tests showed that the recognition of "Disgusted" was impaired when displaying the Wave-like motion $(\mathrm{M}=.82$; $\mathrm{SD}=.39)$ compared to displaying the Translational one $(\mathrm{M}=.95$; $\mathrm{SD}$ $=.22$ ), $\mathrm{p}<.01$. Another finding that got along with our hypotheses was that "Fearful" was more recognized when displaying the Parabolic motion $(\mathrm{M}=.57$; $\mathrm{SD}=.50)$ than when displaying the Wave-like motion $(\mathrm{M}=.46$; $\mathrm{SD}=.50), p<.05$. Both results emphasized the expected positive effects of the Wave-like motion by diminishing the recognition of negative emotional faces. Hence, we can infer that the Wave-like motion impaired the processing of negative faces. (see Table 2).

\section{Perceived Intensity}

The analysis revealed a significant main effect of Emotional Face, $F(5,300)=33.65 ; p<.0001$. It indicated that "Surprised" $(M=6.33$; $S D=1.55)$, "Happy” $(M=6.72 ; S D=1.50)$ and "Disgusted" ( $M=6.88 ; S D=1.59)$ did not differ from one another. Besides, "Angry” ( $M=5.69 ; S D=1.59)$ was perceived as less intense than "Happy", $p<.01$, and "Disgusted", $p<.001$, but as more intense than "Sad", $p<.01$. In fact, the "Sad" expression differed from all other emotional faces, making it the least intensely perceived ( $M=4.72 ; S D=1.67)$. The second least intensely perceived emotional face was "Fearful" $(M=$ 5.42; $S D=1.74$ ), though its score still was higher than "Sad", $p$ $<.05$, and did not significantly differ from “Angry”.

No main effect for the Motion Pattern factor was found.

Nevertheless, the Motion Pattern $\times$ Emotional Face interacttion was significant, $F(10,600)=3.45 ; p<.001$ (see Table 3).

Post-hoc tests indicated that "Surprised" was perceived as more intense when displaying the Wave-like motion than when displaying the Translational one, $p<.01$, or the Parabolic one, $p<.01$. The "Sad" expression was evaluated as more intense when displaying the Parabolic motion than when displaying the Translational motion, $p<.01$. The expected difference between the Wave-like and Para bolic motion were obtained for "Happy" and "Angry", respectively, the Wave-like motion increased the perceived intensity of the "Happy" face, $p<.05$, and decreased the perceived intensity of the "Angry" face, $p<.05$.

Table 1.

Arousal means and standard deviations for the Motion Pattern $\times$ Emotional Face interaction.

\begin{tabular}{ccccccc}
\hline \multicolumn{7}{c}{ Emotion Face } \\
\hline Motion & Surprised & Angry & Happy & Sad & Disgusted & Fearful \\
Translation & $2.92(1.75)$ & $3.25(1.82)$ & $3.20(2.09)$ & $2.97(1.66)$ & $3.36(1.59)$ & $3.16(1.67)$ \\
Parabol & $3.03(1.74)$ & $3.23(1.94)$ & $3.21(1.95)$ & $2.53(1.46)$ & $3.31(1.93)$ & $3.29(1.81)$ \\
Wave & $3.31(2.04)$ & $3.36(1.96)$ & $3.71(2.11)$ & $2.92(1.52)$ & $3.46(1.88)$ & $3.25(1.98)$ \\
\hline
\end{tabular}

Table 2.

Recognition means and standard deviations for the Motion Pattern $\times$ Emotional Face interaction.

\begin{tabular}{cccccccc}
\hline & \multicolumn{5}{c}{ Emotion Face } \\
\hline Motion & Surprised & Angry & Happy & Sad & Disgusted & Fearful \\
Translation & $.88(.32)$ & $.90(.30)$ & $.98(.13)$ & $.70(.46)$ & $.95(.22)$ & $.49(.50)$ \\
Parabol & $.85(.36)$ & $.84(.37)$ & $.98(.13)$ & $.79(.41)$ & $.92(.28)$ & $.57(.50)$ \\
Wave & $.90(.30)$ & $.85(.36)$ & $.97(.18)$ & $.74(.44)$ & $.82(.39)$ & $.46(.50)$ \\
\hline
\end{tabular}

Table 3.

Perceived Intensity means and standard deviations for the Motion Pattern $\times$ Emotional Face interaction.

\begin{tabular}{ccccccc}
\hline & \multicolumn{5}{c}{ Emotion Face } \\
\hline Motion & Surprised & Angry & Happy & Sad & Disgusted & Fearful \\
Translation & $6.15(1.60)$ & $5.72(1.54)$ & $6.77(1.48)$ & $4.39(1.69)$ & $7.02(1.57)$ & $5.52(1.70)$ \\
Parabol & $6.13(1.62)$ & $5.93(1.60)$ & $6.46(1.60)$ & $5.06(1.51)$ & $6.73(1.58)$ & $5.25(1.97)$ \\
Wave & $6.70(1.43)$ & $5.42(1.62)$ & $6.93(1.42)$ & $4.72(1.81)$ & $6.88(1.61)$ & $5.49(1.56)$ \\
\hline
\end{tabular}


The same results were obtained when performing simple effects for each Emotional Face taken alone in the Emotional Face $\times$ Motion Pattern interaction. Motion patterns affected the perceived intensity for three types of emotional faces: "Surprised”, F $(2,120)=5.64 ; p<.01$, "Happy”, F $(2,120)=4.28$; $p<.02$, and "Sad", F $(2,120)=5.84 ; p<.01$. The Wave-like motion increased the perceived intensity of the "Surprised" emotional face compared to the Translational motion and the Parabolic one. In line with the previous result, the Wave-like motion also increased the perceived intensity of the "Happy" face unlike the Translational and the Parabolic motion. Results highlighted that the Parabolic motion increased the perceived intensity of the "Sad" emotional face contrary to the Wave-like and the Translational motion. Besides, it seems substantial to note that other simple effects revealed a tendency for "Angry", $F(2,120)=2.71 ; p=.07$. This tendency showed a similar finding to the one concerning the "Sad" expression, namely, the Parabolic motion tended to increase the perceived intensity of the "Angry" face compared to the Translational and the Wavelike motion. Again, the positive effects from the exposure to the Wave-like motion and the negative effects from the exposure to the Parabolic motion were emphasized by our results concerning the perceived intensities of the emotional faces (see Figure 3).

From that standpoint, we can say that Perceived Intensities confirmed the positive effects of the Wave-like motion and the negative effects of the Parabolic motion. Thus, the Wave-like motion increased the intensities of the "Surprised" and "Happy" emotional faces whilst the Parabolic motion rather increased intensities for negative emotional faces, namely, the "Sad" and "Angry" expressions. Further explanations will be given in the Discussion.

\section{Discussion}

The experiment's findings partly confirm the hypotheses concerning the effects of the Wave-like, Parabolic and Translational motion patterns. Indeed, these specific patterns of motion were thought to change the perception of emotional stimuli. Thus, the Wave-like motion, which is believed to be linked to positive emotions (Podevin, 2009; Rusinek, 2009), plays here an important role in the high perceived intensities of positive faces (i.e., "Surprised" and "Happy") according to the Motion Pattern $\times$ Emotional Face Interaction. This interaction also lea- ds us to state that the Parabolic motion increases perceived intensities of "Sad" emotional face. The Translational motion seems to be related to the "Disgusted" and "Fearful" emotional faces if we take a look at the perceived intensities of these facial expressions. Nevertheless, these findings were non-significant both for the "Disgusted" and "Fearful" conditions. Moreover, the Translational motion was less negative than the Parabolic motion and less positive than the Wave-like motion for other emotional faces. This latter result is in line with theoreticcal assumptions. Another odd finding concerns the high perceived intensities for "Fearful", "Disgusted", and "Sad" faces when displayed with the Wave-like motion, yet believed to be linked to positive faces only. In fact, the only significant and relatively high perceived intensity of that motion with a negative face was found for the Wave-like/“Sad" association. In fact, the Parabolic motion increased more the perceived intensity of the "Sad" face compared to the Wave-like motion. These findings confirm the relationship between positive emotions and Wave-like motion observed by Podevin (2009), but in the mean time, present findings do clearly demonstrate the relationship between negative emotions and Parabolic motion. If Podevin (2009) showed a relationship between emotional words and the three motion patterns, namely, a relation between a visual perception and the verbal domain, results from the present study show that this bond between emotion and motion go far beyond, as emotional faces are non-verbal stimuli. Concerning results such as the high perceived intensity of the "Sad" face displaying the Wave-like motion, an explanation holds in the counterregulation principle (Rothermund, 2003; Rothermund, Voss \& Wentura, 2008; Wentura, Voss, \& Rothermund, 2009). The counter-regulation hypothesis consists in the automatic attention shi$\mathrm{ft}$ towards information that is incongruent with an individual's current emotional dynamics. That principle would therefore permit the individual to be flexible and adaptable to its environment while preventing him from emotional escalation. We could imagine that when watching a "Sad" face displaying the Wave-like motion pattern, the individuals paid more attention to the features of the face and less to the Motion than for any other Emotional Face. Indeed, the "Sad" expression had a particular status as it was the least intensely perceived in our experiment. It is noteworthy that we retrieved results from Goeleven et al. (2008), namely, independently of the motion pattern displayed, "Disgusted", "Happy" and "Surprised" were perceived as the most intense emotional faces.
SURPRISED

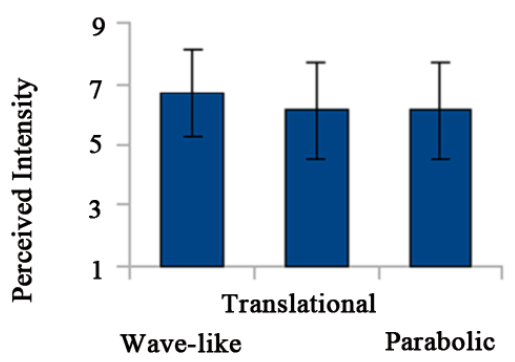

HAPPY

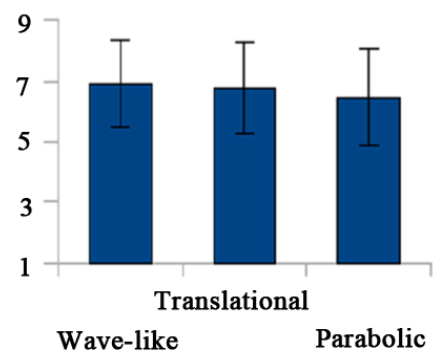

SAD

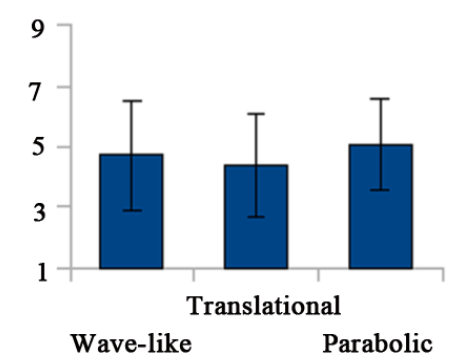

Motion Pattern

Figure 3.

Perceived Intensity means and standard deviations for "Surprised”, "Happy” and "Sad” facial expressions. 
In addition, the Motion Pattern $\times$ Emotional Face interaction results reinforce the idea that the Wave-like motion should be positively evaluated whereas the Parabolic motion should be rather negatively evaluated (Podevin, 2009). That schema of findings seems to be retrieved for the Arousal dimension. Effectively, the Wave-like motion was more arousing when associated to the "Happy" face than both the Parabolic motion and the Translational one. That latter is definitely in accordance with Podevin's works (2009). In line with present findings concerning the Perceived Intensity, another counter-intuitive result was found for the "Sad" expression regarding the Arousal dimension: the Translational motion increased the self-assessed Arousal compared to the Parabolic motion for that emotional face. Again, this would suggest a particular status for the "Sad" emotional expression.

The lack of arousing power combined to the increase of negative emotions' perceived intensities for the Parabolic motion could help explaining his harmful effects on a cognitive task in Podevin (2009). Likewise, the arousing power of the Wave-like motion combined to its emotional perceived intensity features can account for the drawing of smiles to a plain black disk performing that pattern of motion (Podevin, 2009). It is very plausible that high arousal levels associated to the experiment's task could be taken as a positive marker. Indeed, the fact that the task is somewhat interactive should involve participants' interest. Therefore, a weak level of arousal could highlight that the participant showed little interest to the task while a strong level of arousal could trace the interest of the participant (Simons, Detenber, Roedema, \& Reiss, 1999; Simons, Detenber, Reiss, \& Shults, 2000). By contrast, the exact effects implied by the Translational motion still has to be questioned. Indeed, Phaf and Rotteveel (2009) showed that a translational arrow going from left to right is even more arousing and more positive than emotional faces. These authors proved the positivity of a left-toright arrow in an explicit manner (i.e., subjective self-reports) and in an implicit manner (i.e., approach vs. avoidance). That latter gives powerful evidence to the positive involvements of left-to-right monitoring in left-to-right readers and therefore, we could have expected the Translational motion to be evaluated more positively than the Wave-like motion and the Parabolic motion. In effect, the Translational motion was the only pattern that was smooth and uninterrupted, while both others were jerky.

The "Happy" face occurs to be more recognized than the "Sad" and "Fearful" expressions, which makes it the most recognized emotional face in the experiment. This result is in line with what was shown under an explicit perception condition by Calvo and Lundqvist (2008). Also, "Fearful” was the least recognized emotional expression in the study as in their research. The Wave-like motion impaired the recognition of "Disgusted" compared to the Translational motion whilst the Parabolic motion increased the recognition of "Fearful" compared to the Wave-like motion. Both findings reinforce the assumption that the Wave-like motion is associated to positive emotional stimuli whereas the Parabolic motion is associated to negative emotional stimuli.

\section{Conclusion}

In sum, the present study could be seen as a successful attempt to investigate the three specific patterns of motion which are somehow linked to emotions, especially discrete emotions.
Thus, Ekman evolved from a vision of emotion that is global (i.e., positive vs. negative) to a discrete vision of emotion (i.e., basic emotions), and the current research used six basic emotional faces in order to stay relevant to this view. Furthermore, the Arousal has also been investigated as it was believed to be a substantial component of emotional experiences. Further research shall be directed towards a more dynamic prospect (i.e., emotional films, emotional scenes) and more ecological environment so that one quietly understands the effects of these patterns of motion, especially their parts in the affective monitoring. Effectively, the main limitation of the present study holds in the lack of ecological validity as one could expect faces to be moving in their inner properties (e.g., emotional faces videos) rather than in their whole. Another prospect concerns the use of motion in cognitive-behavioral therapies such as Rusinek (2009) suggested it in his trial to desensitize sub-clinical arachnophobia. A final aspect of the motion-emotion link research would lay in Sports and their many applications. For example, it is now proved that Taekwondo distinguishes between four types of motion. Hence, it would be possible to investigate the effects of the three above-studied motions in martial artists or other sportsmen/women.

\section{Acknowledgements}

The research reported in this article has been made possible thanks to the Dr. Gaëlle Podevin and Dr. Céline Douilliez who provided us most of our material.

\section{REFERENCES}

Adolphs, R. (2002). Recognizing emotion from facial expressions: Psychological and neurological mechanisms. Behavioral and Cognitive Neuroscience Review, 1, 21-62.

Alexopulos, T., \& Ric, F. (2007). The Evaluation-behavior link: Direct and beyond valence. Journal of Experimental Social Psychology, 43, 1010-1016. doi:10.1016/j.jesp.2006.10.017

Ambadar, Z., Schooler, J. W., \& Cohn, J. F. (2005). Deciphering the enigmatic face: The importance of facial dynamics in interpreting subtle facial expressions. Psychological Science, 16, 403-410. doi:10.1111/j.0956-7976.2005.01548.x

Barsalou, L. W. (1999). Perceptual symbol systems. Behavioral and Brain Sciences, 22, 577-660.

Batty, M. \& Taylor, M. J. (2003). Early processing of the six basic emotional expressions. Cognitive Brain Research, 17, 613-620. doi:10.1016/S0926-6410(03)00174-5

Beaupré, M. G., Cheung, N., \& Hess, U. (2000). The Montreal set of facial displays of emotion [Slides]. Montreal, Canada: Department of Psychology, University of Quebec.

Berridge, K. C. (2003). Pleasures of the brain. Brain and Cognition, 52, 106-128. doi:10.1016/S0278-2626(03)00014-9

Blake, R., \& Shiffrar, M. (2007). Perception of human motion. Annual Review of Psychology, 58, 47-74. doi:10.1146/annurev.psych.57.102904.190152

Brouillet, T., Heurley, L., Martin, S., \& Brouillet, D. (2010). The embodied cognition theory and the motor component of "yes" and "no" verbal responses. Acta Psychologica, 134, 310-317. doi:10.1016/j.actpsy.2010.03.003

Calvo, M. G., \& Lundqvist, D. (2008). Facial expressions of Emotion (KDEF): Identification under different display-duration conditions. Behavior Research Methods, 40, 109-115. doi:10.3758/BRM.40.1.109

Casasanto, D., \& Dijkstra, K. (2010). Motor Action and Emotional Memory. Cognition, 115, 179-185. doi:10.1016/j.cognition.2009.11.002

Chen, M., \& Bargh, J. A. (1999). Consequences of automatic eva- 
luation: Immediate behavioral predispositions to approach or avoid the stimulus. Personnality and Social Psychology Bulletin, 25, 215224. doi:10.1177/0146167299025002007

Creem, S. H., \& Proffitt, D. R. (2001). Defining the cortical visual systems: "What", "Where" and "How". Acta Psychologica, 107, 43-68. doi:10.1016/S0001-6918(01)00021-X

Crockford, D. N., Goodyear, B., Edwards, J., Quickfall, J., \& el-Guebaly, N. (2005). Cue-induced brain activity in pathological gamblers. Biological Psychiatry, 58, 787-795. doi:10.1016/j.biopsych.2005.04.037

Cutting, J. E., \& Kozlowski, L. T. (1977). Recognizing friends by their walk: Gait perception without familiarity cues. Bulletin of the Psychonomic Society, 9, 353-356.

Detenber, B. H., Simons, R. F., \& Bennet, G. C. (1998). Roll’ Em: The effects of picture motion on emotional responses. Journal of Broadcasting \& Electronic Media, 42, 112-126. doi:10.1080/08838159809364437

Ekman, P. (1993). Facial expression and emotion. American Psychologist, 48, 384-392. doi:10.1037/0003-066X.48.4.384

Ekman, P. (1999). Facial expressions. In Dalgleish, T., \& Power, M. (Eds.), Handbook of cognition and emotion (pp. 301-320). New York: John Wiley \& Sons Ltd.

Ekman, P., \& Friesen, W. V. (1976). Measuring facial movement. Environmental Psychology and Nonverbal Behavior, 1, 56-75. doi:10.1007/BF01115465

Ekman, P., Friesen, W. V., \& Tomkins, S. S. (1971). Facial affect scoring technique: A first validity study. Semiotica, 3, 37-58. doi:10.1515/semi.1971.3.1.37

Freina, L., Baroni, G., Borghi, A. M., \& Nicoletti, R. (2009). Emotive-concept nouns and motor responses: Attraction or repulsion? Memory \& Cognition, 37, 493-499. doi:10.3758/MC.37.4.493

Goeleven, E., DeRaedt, R., Leyman, L., \& Verschuere, B. (2008). The Karolinska directed emotional faces: A validation study. Cognition and Emotion, 22, 1094-1118. doi:10.1080/02699930701626582

Goodale, M. A., \& Milner, A. D. (1992). Separate visual pathways for perception and action. Trends in Neurosciences, 15, 21-26. doi:10.1016/0166-2236(92)90344-8

Gross, J. J., \& Levenson, R. W. (1995). Emotion elicitation using films. Cognition and Emotion, 9, 87-108. doi:10.1080/02699939508408966

Heider, F., \& Simmel, M. (1944). An experimental study of apparent behavior. American Journal of Psychology, 57, 243-259. doi:10.2307/1416950

Johansson, G. (1973). Visual perception of biological motion and a model for its analysis. Perception and Psychophysics, 14, 202-211. doi:10.3758/BF03212378

Lang, P. J. (1980). Behavioral treatment and bio-behavioral assessment. In J. B. Sidowski, J. H. Johnson, \& T. A. Williams (Eds.), Technology in mental health care delivery systems (pp. 119-167). Norwood, NY: Ablex. doi:10.1111/j.1469-8986.1993.tb03352.x

Lang, P. J., Greenwald, M. K., Bradley, M. M. \& Hamm, A. O. (1993). Looking at pictures: Affective, facial, visceral and behavioral reactions. Psychophysiology, 30, 261-273.

Lundqvist, D., Flykt, A., \& Öhman, A. (1998). The karolinska directed emotional face (KDEF). Stockholm: Department of Neurosciences Karolinska Hospital.

Matsumoto, D., \& Ekman, P. (1988). Japanese and Caucasion Facial Expressions of Emotion (JACFEE) [Slides]. San Francisco: Intercultural and Emotion Research Laboratory, Department of Psychology, San Francisco State University.

Méary, D., Kitromilides, E., Mazens, K., Graff, C., \& Gentaz, E. (2007). Four-day-old human neonates look longer at non-biological motions of a single point-of-light. Plos ONE, 2, 186. doi:10.1371/journal.pone.0000186

Michotte, A. (1946). La perception de la causalité. Louvain: Publications Universitaires de Louvain; Paris-Bruxelles: Erasme; AnversGand-Amsterdam: Standaard-Boekhandel.

Michotte, A. (1962). Causalité, permanence et réalité phénoménales. Louvain: Publications Universitaires de Louvain.

Morris, J. S., DeGelder, B., Weiskrantz, L., \& Dolan, R. J. (2001). Differential extrageniculostriate and amygdala responses to presentation of emotional faces in a cortically blind field. Brain, 124, 1241-1252. doi:10.1093/brain/124.6.1241

Phaf, R. H., \& Rotteveel, M. (2005). Affective modulation of recognition bias. Emotion, 5, 309-318. doi:10.1037/1528-3542.5.3.309

Phaf, R. H., \& Rotteveel, M. (2009). Looking at the bright side: The affective monitoring of direction. Emotion, 9, 729-733. doi:10.1037/a0016308

Podevin, G. (2009). Influence des mouvements elementaires sur les processus cognitifs emotionnels. Thèse de Doctorat, Université de Lille Nord de France UDL3.

Rimé, B., Boulanger, B., Laubin, P., Richir, M., \& Stroobants, K. (1985). The perception of interpersonal emotions originated by patterns of movement. Motivation and Emotion, 9, 241-260. doi:10.1007/BF00991830

Rimé, B., \& Schiaratura, L. (1991). Gesture and speech. In R. S. Feldman, \& B. Rimé (Eds.), Fundamentals of nonverbal behavior (pp. 239-281). New York: Press Syndicate of the University of Cambridge.

Rothermund, K. (2003). Motivation and attention: Incongruent effects of feedback on the processing of valence. Emotion, 3, 223-238. doi:10.1037/1528-3542.3.3.223

Rothermund, K., Voss, A., \& Wentura, D. (2008). Counter-regulation in affective attentional biases: A basic mechanism that warrants flexibility in emotion and motivation. Emotion, 8, 34-46. doi:10.1037/1528-3542.8.1.34

Rusinek, S. (2009). Désensibilisation de l'arachnophobie à l'aide d'une induction émotionnelle positive par le mouvement. 1er Congrès Français de Psychiatrie, Nice, décembre 2009.

Schaefer, A., Nils, F., Sanchez, X., \& Philippot, P. (2010). Assessing the effectiveness of a large database of emotion-eliciting films: A new tool for emotion researchers. Cognition and Emotion, 24, 11531172. doi:10.1080/02699930903274322

Simons, R. F., Detenber, B. H., Roedema, T. M., \& Reiss, J. E. (1999). Emotion processing in three systems: The medium and the message. Psychophysiology, 36, 619-627. doi:10.1111/1469-8986.3650619

Simons, R. F., Detenber, B. H., Reiss, J. E., \& Shults, C. W. (2000). Image motion and context: A between and within subjects com- parison. Psychophysiology, 37, 706-710. doi:10.1111/1469-8986.3750706

Tremoulet, P. D., \& Feldman, J. (2000). Perception of animacy from the motion of a single object. Perception, 29, 943-951. doi:10.1068/p3101

Troje, N. F., Westhoff, C., \& Lavrov, M. (2005). Person identification from biological motion: Effects of structural and kinematic cues. Perception \& Psychophysics, 67, 667-675. doi:10.3758/BF03193523

Troje, N. F., \& Westhoff, C. (2006). The inversion effect in biological motion perception: Evidence for a "Life Detector"? Current Biology, 16, 821-824. doi:10.1016/j.cub.2006.03.022

Wentura, D., Voss, A., \& Rothermund, K. (2009). Playing TETRIS for science counter-regulatory affective processing in a motivationnally "hot" context. Acta Psychologica, 131, 171-177.

doi:10.1016/j.actpsy.2009.05.008 